\title{
VARIATIONAL TRANSITION STATE THEORY
}

\author{
Donald G. Truhlar \\ Department of Chemistry, University of Minnesota \\ Minneapolis, MN 55455-0431
}

$\mathrm{DOE} / \mathrm{ER} / 13579--22$

\section{Introduction}

DE91 001253

In recent years our research group has made a systematic effort to study the validity of transition state theory (TST). We have found that the conventional theory is sometimes remarkably accurate, but in many other cases it leads to large errors. Fortunately we have found that a much more reliable theory that has many of the advantages of conventional TST can also be formulated, and it can be applied to practical problems with an effort that is much closer to that required for conventional transition state theory than to that required for full quantal dynamics calculations. The two most important features in the improved approach to transition state theory are the variational determination of the transition state and the incorporation of tunneling contributions by multidimensional semiclassical approximations.

\section{Scope of project}

This project is concerned with the development and application of generalized TST and multidimensional semiclassical tunneling approximations to chemical reaction rates. We have developed and implemented several practical versions of variational transition state theory (VTST), namely, canonical variational theory (CVT), improved canonical variational theory (ICVT), and microcanonical variational theory $(\mu \mathrm{VTT})$. We have also developed and implemented several multidimensional semiclassical tunneling approximations, the most accurate and useful of which are the small-curvature semiclassical adiabatic (SCSA), largecurvature version-3 (LC3), and least-action (LA) approximations. We have applied the methods to theimal rate constants, using transmission coefficients based on ground-state tunneling, and we have also presented and applied adiabatic and diabatic extensions to calculate rate constants for vibrationally excited reactants. Our general goal is to develop accurate method for calculating chemical reaction rate constants that remain practical even for reasonably complicated molecules. The approximations mentioned above yield rate constants for systems whose potential energy surface is known or assumed. A second, equally important aspect of our work is the determination or modeling, semiempirically and/or from electronic structure calculations, of potential energy surfaces. In this regard we have developed and implemented new flexible procedures for representing the potential surfaces of reactive systems by global and semiglobal analytic functions, and we have used our techniques to develop more accurate potential energy surfaces for several systems. A new initiative is concerned with the development of algorithms to calculate rate constants based on a minimum of information about the potential energy surface.

\section{Recent results}

We have developed a general computer program, called POLYRATE, for calculating the rate constants of gas-phase, solid-state, or gas-solid-interface chemical reactions involving one or two reactants and any number of atoms, and we have made this available in an international program library with 20 test runs and a 204-page program manual. (An update, called version 1.6.2, is also available.) The POLYRATE computer program accepts information about the potential energy surface in the form of either an analytic potential energy function or a seciuence of geometries, energies, gradients, and second (or higher)

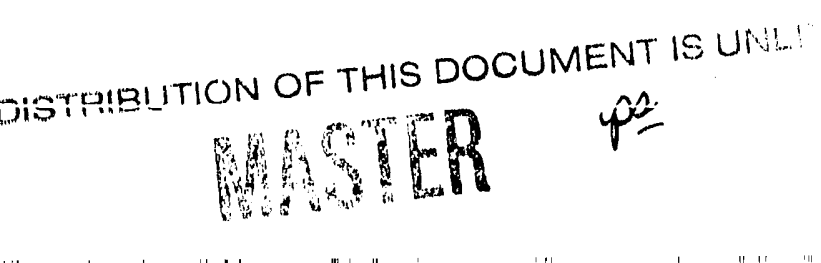


derivative matrices at points along the reaction path. The program calculates the rate constant for quantized internal degrees of freedom and classical reaction-path motion by VTST. The probabilities for tunneling and nonclassical reflection are estimated by semiclassical methods and incorporated by a transmission coefficient, which for thermal reactions is based on the ground state.

We have applied the methods of the POLYRATE program to calibrate a sequence of three successively improved new semiempirical potential energy surfaces for the reaction $\mathrm{CH}_{3}+$ $\mathrm{H}_{2} \rightarrow \mathrm{CH}_{4}+\mathrm{H}$. The calibration was based on ab initio electronic structure calculations and experimental thermochemical data, vibrational frequencies, reaction rate constants, Arrhenius parameters, and $\mathrm{H}_{2} / \mathrm{D}_{2}$ kinetic isotope effects (KIEs); it used ICVT calculations with SCSA ground-state transmission coefficients (ICVT/SCSAG) to compare to the experimental kinetic data. The best surface yields rate constants for the forward and reverse reaction, activation energies, and $\mathrm{H}_{2} / \mathrm{D}_{2} \mathrm{KIE}$ 's that are consistent with most of the available experimental data.

One of these new $\mathrm{CH}_{5}$ potential energy surfaces has been used for the first application of the LC3 tunneling method to a polyatomic reaction. Tunneling contributions to thermal reaction rates for abstraction of $\mathrm{H}$ or $\mathrm{D}$ from $\mathrm{H}_{2}, \mathrm{HD}, \mathrm{D}_{2}$, or a model of benzene were calculated. We found encouragingly similar results to those obtained by the SCSA method for abstraction of $\mathrm{H}$ from $\mathrm{H}_{2}$ or $\mathrm{D}$ from $\mathrm{HD}$ or $\mathrm{D}_{2}$, but-as expected-the LC3 method predicts much more tunneling than the SCSA one for the other cases. The LC 3 tunneling method slightly improves the agreement with experiment for the internivlecular $\mathrm{H}_{2} / \mathrm{D}_{2}$ kinetic isotope effects, and this lends added credibility to the potential energy surface we created. The results for HD indicate that the intramolecular kinetic isotope effect provides a very critical test of theory. The results for abstraction from benzene show, as also found in previous work, that tunneling methods based on minimum energy reaction paths or tunneling paths close to or based on such paths seriously underestimate the amount of tunneling for the transfer of a light atom between two heavy moieties.

The new $\mathrm{CH}_{5}$ potential energy surfaces and the SCSA and LC3 tunneling methods have also been used with variational transition state theory to study secondary $\alpha$-deuterium kinetic isotope effects in the reactions $\mathrm{H}+\mathrm{CH}_{4} / \mathrm{CHD}_{3} \leftrightarrow \mathrm{H}_{2}+\mathrm{C}_{2} \mathrm{I}_{3} / \mathrm{CD}_{3}$ and $\mathrm{D}+$ $\mathrm{CH}_{4} / \mathrm{CHD}_{3} \rightarrow \mathrm{HD}+\mathrm{CH}_{3} / \mathrm{CD}_{3}$. We found that both variational optimization of the transition state and tunneling have large effects on these kinetic isotope effects. For example for $\mathrm{H}+\mathrm{CH}_{4} / \mathrm{CHCD}_{3} \rightarrow \mathrm{H}_{2}+\mathrm{CH}_{3} / \mathrm{CD}_{3}$, variational optimization of the transition state increases the deviation of kinetic isotope effert from unity by a factor of 3 , and tunneling increases this deviation by a further factor of 2 . This has important implications for the widespread use of secondary deuterium kinetic isotope effects for mechanistic analysis.

Most recently we have completed a detailed study of the gas-phase $\mathrm{S}_{\mathrm{N}} 2$ reaction $\mathrm{Cl}^{-}(\mathrm{g})+\mathrm{CH}_{3} \mathrm{Cl}^{\prime}(\mathrm{g}) \rightarrow \mathrm{CH}_{3} \mathrm{Cl}(\mathrm{g})+\mathrm{Cl}^{\prime-}(\mathrm{g})$ as well as the microhydrated cluster reactions $\mathrm{Cl}^{-}\left(\mathrm{H}_{2} \mathrm{O}\right)_{n}(\mathrm{~g})+\mathrm{CH}_{3} \mathrm{Cl}^{\prime}(\mathrm{g}) \rightarrow \mathrm{CH}_{3} \mathrm{Cl}(\mathrm{g})+\mathrm{Cl}^{\prime}\left(\mathrm{H}_{2} \mathrm{O}\right)_{\mathrm{n}}(\mathrm{g})$, with $\mathrm{n}=1$ and 2. These reactions involve up to 36 degrees of freedom. The case of $n=1$ was used to test theories of nonequilibrium solvation. For example, although the attachment of one water molecule to the anion lowers the reaction rate by a factor of 3200 at room temperature, we found that variational transition state theory could be used to optimize an equilibrium solvation model that accounts for the effect within $11 \%$.

We have also reported a test of the LA tunneling approximation against accurate quantum dynamics calculations for the reaction $\mathrm{O}+\mathrm{HD} \rightarrow \mathrm{OH}+\mathrm{D}$ and $\mathrm{OD}+\mathrm{H}$. The semiclassical and accurate quantal results are in excellent agreement, and, in particular, the 
semiclassical results agree with the accurate quantal ones in showing a crossover such that the more favored product changes from OD to $\mathrm{OH}$ as the energy is increased. This bodes well for the reliability of our predictions of kinetic isotope effects on other systems where accurate quantum dynamics calculations are not feasible.

\section{Future plans}

We plan to continue our studies involving VTST and semiclassical tunneling calculations for gas-phase chemical reactions. The long-term framework that guides the present research project is as follows: This project is directed to the treatment of chemical reaction rate processes by methods that utilize transition state bottleneck properties and semiclassical methods to incorporate quantal effecis. I am especially interested in utilizing these methods to incorporate quantal effects and to predict total reaction rates without explicitly averaging over completely detailed state-to-state rate constants. The fact that transition state theory and its generalizations can often address this computational task successfully in terms of localized dynamical bottlenecks has long been one of its strengths. In the first years of this project we implemented a successful variational version of transition state theory and new, general methods for incorporating multidimensional effects in semiclassical transmission coefficients, and we demonstrated the reliability of these methods. We are now in the process of improving the implementation and extending it to a wider variety of gas-phase rate processes, including atom transfers, general metatheses, isomerizations, dissociations, and association reactions. Our choices of individual reactions are guided by weighing the fundamental interest of a given reaction and perhaps its value as a prototype for a class of reactions, as well as-in some cases-by the importance of a reaction or reaction type for combustion or for the knowledge base on which other existing or future energy programs can evolve.

Two approaches to the calculation of reaction rates from limited information about the potential energy surface are being developed. In the first approach, direct dynamics, the rate constant is calculated directly from electronic structure input without any intermediate fitting. In the second, interpolated variational transition state theory, a reaction path and the potential expanded around a reaction valley are obtained by analytic representations using appropriate functional forms with parameters fitted to electronic structure calculations at a limited number of points.

We are also incorporating more accurate reaction-path following and the LC3 tunneling method into a new distribution version (2.0) of the POLYRATE computer code.

\section{Publications 1988-early 1990}

1. "Algorithms and Accuracy Requirements for Computing Reaction Paths by the Method of Steepest Descent," B. C. Garrett, M. J. Redmon, R. Steckler, D. G. Truhlar, K. K. Baldridge, D. Bartol, M. W. Schmidt, and M. S. Gordon, Journal of Physical Chemistry 92, 1476-1488 (1988).

2. "Improved Potential Energy Surfaces for the Reaction $\mathrm{O}\left({ }^{3} \mathrm{P}\right)+\mathrm{H}_{2} \rightarrow \mathrm{OH}+\mathrm{H}$," $\mathrm{T}$. Joseph, D. G. Truhlar, and B. C. Garrett, Journal of Chemical Physics 88, 69826990 (1988). 
3. "Thermochemical Kinetic Analysis of Tunneling and the Incorporation of Tunneling Contributions in Thermochemical Kinetics," D. G. Truhlar and B. C. Garrett, Journal of the American Chemical Society 111, 1232-1236 (1989).

4. "Reaction-Path Analysis of the Effect of Monomer Excitation on the Tunneling Splitting of Hydrogen Fluoride Dimer," G. C. Hancock and D. G. Truhlar, Journal of Chemical Physics 90, 3498-3505 (1989).

5. "Dynamical Formulation of Transition State Theory: Variational Transition States and Semiclassical Tunneling," S. C. Tucker and D. G. Truhlar, in New Theoretical Concepts for Understanding Organic Reactions, edited by J. Ber'tán and I. G. Czismadia (Kluwer Academic Publisher, Dordrect , 1989), pp. 291-346.

6. "Test of the Accuracy of Small-Curvature and Minimum-Energy Reference Paths for Parameterizing the Search for Least-Action Tunneling Paths: $(\mathrm{H}, \mathrm{D})+\mathrm{H} \mathrm{Br} \rightarrow$ $(\mathrm{H}, \mathrm{D}) \mathrm{Br}+\mathrm{H}^{\prime}, "$ G. C. Lynch, D. G. Truhlar, and B. C. Garrett, Journal of Chemical Physics 90, 3102-3109 (1989).

7. "Global Potential Energy Surfaces for $\mathrm{H}_{2} \mathrm{Cl}$," D. W. Schwenke, S. C. Tucker, R. Steckler, F. B. Brown, G. C. Lynch, D. G. Truhlar, and B. C. Garrett, Journal of Chemical Physics 90, 3110-3120 (1989).

8. "POLYRATE Program Manual, Version 1.5," A. D. Isaacson, D. G. Truhlar, S. N. Rai, G. C. Hancock, J. G. Lauderdale, T. N. Truong, T. Joseph, B. C. Garrett, and R. Steckler, University of Minnesota Supercomputer Institute Research Report UMSI88/87, September 1988. Copies may obtained by writing to Research Reports Coordinator, Minnesota Supercomputer Institute, University of Minnesota, 1200 Washington Avenue South, Minneapolis, MN 55415.

9. "Ab Initio Reaction Paths and Direct Dynamics Calculations," K. K. Baldridge, M. S. Gordon, R. Steckler, and D. G. Truhlar, Journal of Physical Chemistry 93, 5107-5119 (1989).

10. "Semiclassical and Quantum Mechanical Calculations of Isotopic Kinetic Branching Ratios for the Reaction of $\mathrm{O}\left({ }^{3} \mathrm{P}\right)$ with $\mathrm{HD}$, " G. C. Lynch, P. Halvick, D. G. Truhlar, B. C. Garrett, D. W. Schwenke, and D. J. Kouri, Zeitschrift für Naturforschung 44a, 427-434 (1989).

11. "Application of the Large-Curvature Tunneling $\wedge$ pproximation to Polyatomic Molecules" Abstraction of H or D by Methyl Radical," B. C. Garrett, T. Joseph, T. N. Truong, and D. G. Truhlar, Chemical Physics 136, 271-284 (1989).

12. "A Six-Body Potential Energy Surface for the $\mathrm{S}_{\mathrm{N}} 2$ Reaction $\mathrm{Cl}^{-}(\mathrm{g})+\mathrm{CH}_{3} \mathrm{Cl}(\mathrm{g})$ and a Variational Transition State Theory Calculation of the Rate Constant," S. C. Tucker and D. G. Iruhlar, Joumal of the American Chemical Society, in press.

13. "The Effect of Nonequilibrium Solvation on Chemical Reaction Rates. Variational Transition State Theory Studies of the Microsolvated Reaction $\mathrm{Cl}^{-}\left(\mathrm{H}_{2} \mathrm{O}\right)_{\mathrm{n}}+\mathrm{CH}_{3} \mathrm{Cl}$," S. C. Tucker and D. G. Truhlar, Journal of the American Chemical Society, in press.

\section{DISCLAIMER}

This report was prepared as an account of work sponsored by an agency of the United States Government. Neither the United States (iuvernment nor any agency thereof, nor any of their employees, makes any warranty, express or implied, or assumes any legal liability or responsibility for the accuracy, completeness, or usefulness of any information, apparatus, product, of process disclosed, or represents that its use would not infringe privately owned rights. Reference herein to any specific commercial presluct, precess, wr service by trade name, trademark. manufialures. or otherwise does not necessiarily constitute or imply its endorsement, recommendation, or favoring by the IInited States Government or any agency thereot. The vesws and opinions of authors expressed herein do not necessarity state or reflect those ar whe United States Government or any agency thereof. 

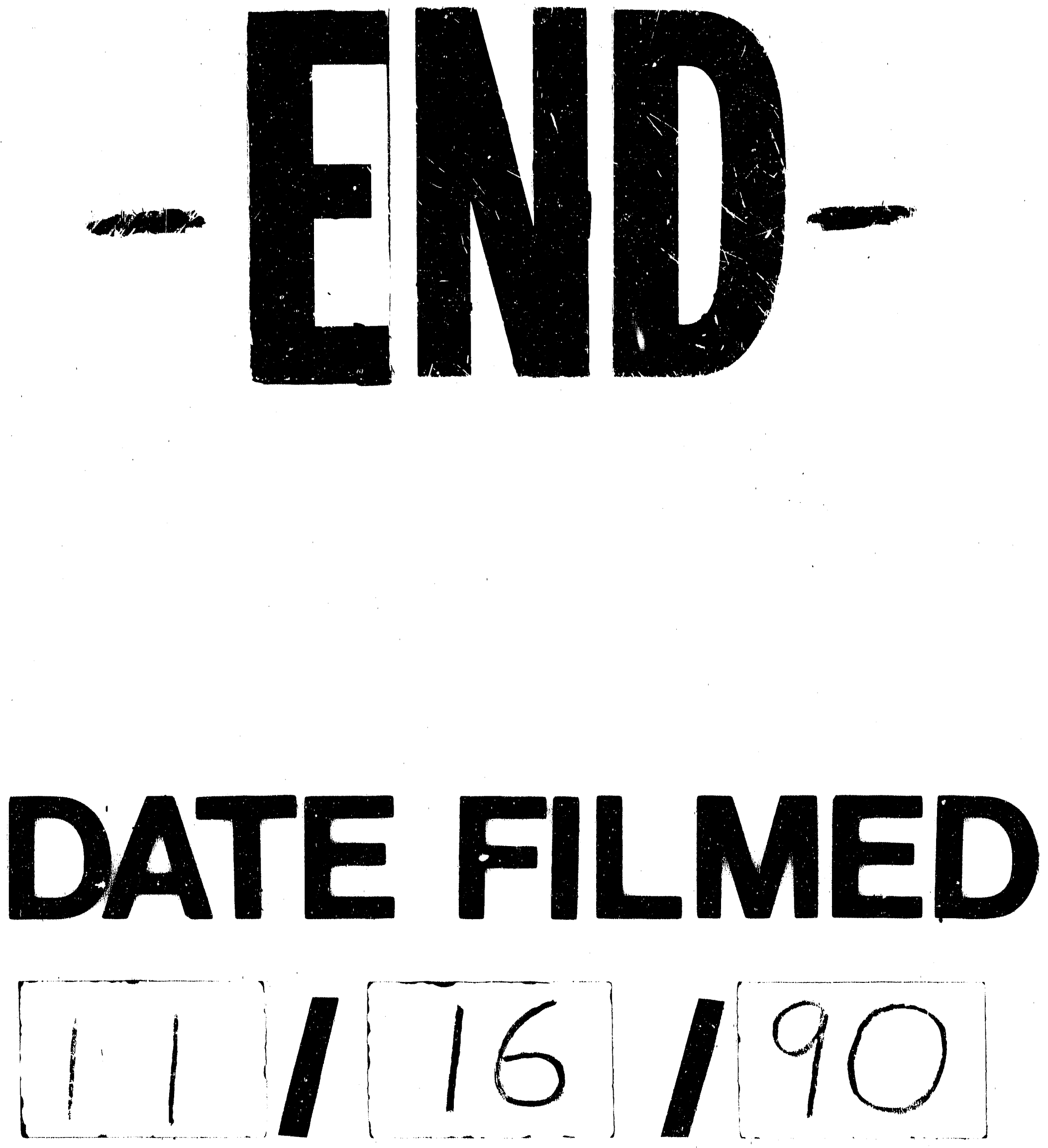
